\title{
TIME-DELAY MODELLING FOR MULTI-LAYER POWER SYSTEMS
}

\author{
I.A. Hiskens \\ Department of Electrical and Computer Engineering \\ University of Wisconsin - Madison \\ 1415 University Drive \\ Madison, WI 53706, USA \\ hiskens@engr.wisc.edu
}

\begin{abstract}
Modelling of multi-layer power system interactions will become increasingly important as market mechanisms and feedback controls become more tightly coupled into the physical system layer. Time-delays are an important aspect of that inter-layer coupling. This paper integrates deterministic, nonlinear time-delays into a systematic hybrid (continuous/discrete) system model. The paper addresses issues arising in the implementation of implicit numerical integration techniques, and computation of trajectory sensitivities. Applications that have the form of inverse problems are motivated.
\end{abstract}

\section{INTRODUCTION}

The traditional view of power system dynamics has focussed primarily on the behaviour of components within the physical system. Little attention has been paid to interactions between the physical system and higher level layers. However with tighter integration of market mechanisms and feedback controls, the modelling of those linkages is becoming increasingly important [1].

A postulated scenario of multi-layer interactions, leading ultimately to cascading failure of the system, is presented in Fig. 1. Whilst time-scale separation currently makes such a scenario unlikely, that comfort zone is steadily shrinking. Time-delays are an important aspect of the interlinking of layers, and play a crucial role in the postulated failure scenario. This paper therefore addresses issues of incorporating time-delays into dynamical models of multi-layer power systems.

Deterministic modelling of power systems provides insights into dynamical behaviour, and underlies most analysis and design functions. Therefore, even though time-delays are usually random, a deterministic (nonlinear) time-delay model has been adopted here. This model can be thought of as describing average response. The model is fully developed, in the context of a systematic hybrid (continu- ous/discrete) system representation, in Section 2. This systematic modelling methodology facilitates algorithms for addressing inverse problems, such as parameter estimation and shooting methods $[2,3]$. Further discussion of applications is provided in Section 5.

\section{MODEL}

\subsection{Extended DAIS model}

Power systems are composed of components that exhibit continuous dynamic behaviour, along with others that respond discretely. Examples of the former include generators and controllers, whilst the latter include switched shunts and controller saturation limits. Furthermore, in multilayer systems, commands are often communicated as discrete signals. Systems that exhibit intrinsic continuous/discrete interactions have become known generically as hybrid systems. Power systems therefore form an important example of this class of system.

It has been shown in $[3,4]$ that hybrid systems can be modelled as a set of differential-algebraic equations, adapted to incorporate impulsive (state reset) action, and switching of the algebraic equations. The importance of time-delay effects on the behaviour of multilayer systems was highlighted in Section 1. Therefore this paper extends that $\underline{D A}$

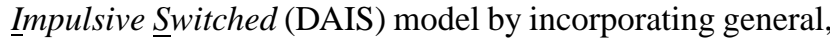
nonlinear time delays. The resulting model has the form

$$
\begin{gathered}
\dot{x}=f(x, y)+\sum_{j=1}^{r} \delta\left(y_{r, j}\right)\left(h_{j}(x, y)-x\right) \\
0=g\left(x, y, y_{d}\right) \equiv g^{(0)}\left(x, y, y_{d}\right)+\sum_{i=1}^{s} g^{(i)}\left(x, y, y_{d}\right) \\
y_{d}(t)=\bar{y}(\theta(y, t))
\end{gathered}
$$

where

$$
g^{(i)}\left(x, y, y_{d}\right)= \begin{cases}g^{(i-)}\left(x, y, y_{d}\right), & y_{s, i}<0 \\ g^{(i+)}\left(x, y, y_{d}\right), & y_{s, i}>0\end{cases}
$$




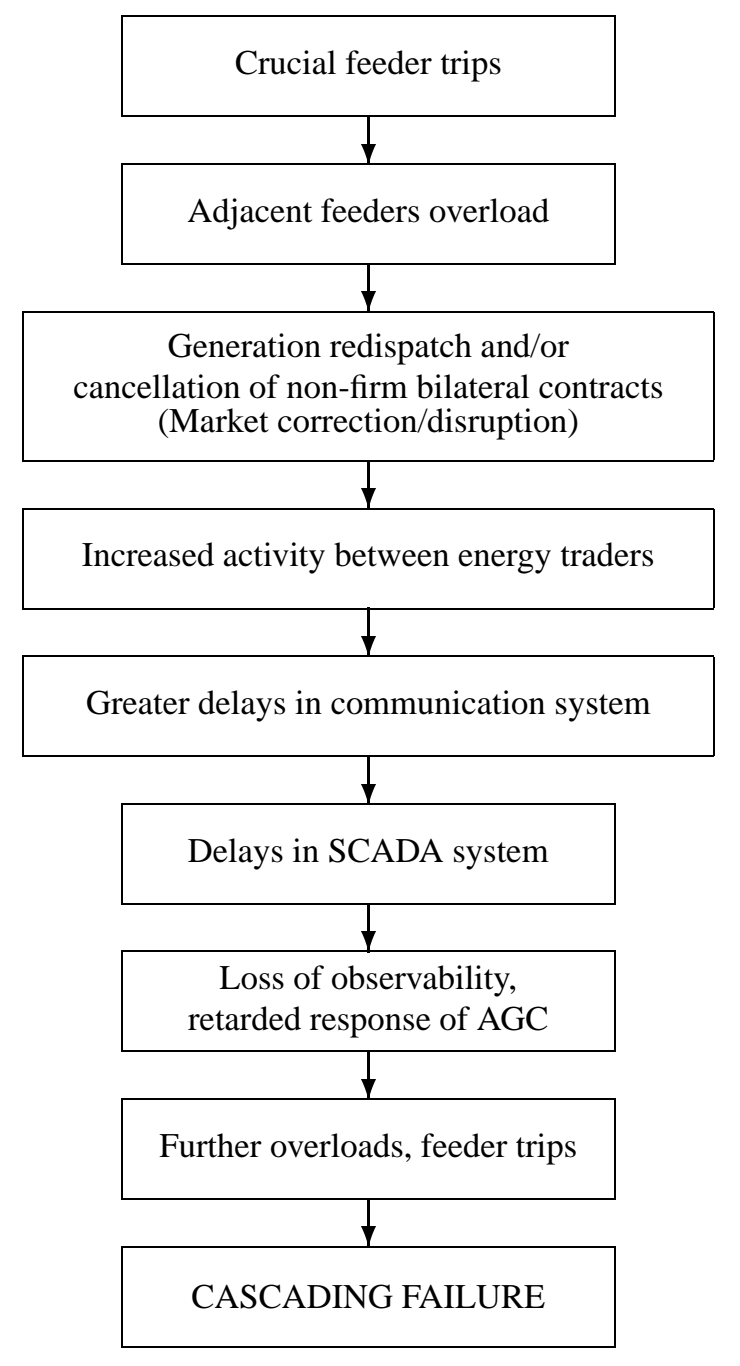

Fig. 1. Market induced cascading failure.

and (3) should be interpreted as

$$
y_{d, i}(t)=\bar{y}_{i}\left(\theta_{i}(y, t)\right)
$$

with the vector $\bar{y}$ composed of selected elements of $y$. Let $\ell$ denote the vector of pointers establishing the correspondance between $\bar{y}$ and $y$. Then

$$
\bar{y}_{[i]}=y_{\left[\ell_{i}\right]}
$$

implies that the $i^{t h}$ element of $\bar{y}$ corresponds to the $\ell_{i}^{t h}$ element of $y$. We shall adopt the vector notation

$$
\bar{y}=y_{[\ell]} .
$$

More than one element of $\bar{y}$ may refer to the same element of $y$. (This would be the case where a component model required the same variable to be delayed by various time periods.)
The impulsive action of state resetting is captured by the Dirac delta $\delta($.$) . The state reset equations can be expressed$ in the alternative form

$$
x^{+}=h_{j}\left(x^{-}, y^{-}\right) \quad \text { when } y_{r, j}=0 .
$$

The flows of the dynamic states $x$ and algebraic states $y$ are defined as

$$
\begin{aligned}
& x(t)=\phi_{x}\left(x_{0}, t\right) \\
& y(t)=\phi_{y}\left(x_{0}, t\right)
\end{aligned}
$$

where $x(t)$ and $y(t)$ satisfy (1)-(4), implying that

$$
\begin{aligned}
y_{d}(t) & =\phi_{\bar{y}}\left(x_{0}, \theta\left(\phi_{y}\left(x_{0}, t\right), t\right)\right) \\
& =\phi_{y_{d}}\left(x_{0}, t\right) .
\end{aligned}
$$

The flows satisfy the initial conditions,

$$
\begin{gathered}
\phi_{x}\left(x_{0}, t_{0}\right)=x_{0} \\
g\left(x_{0}, \phi_{y}\left(x_{0}, t_{0}\right), \phi_{y_{d}}\left(x_{0}, t_{0}\right)\right)=0 .
\end{gathered}
$$

An ambiguity arises in the model when $\theta(y, t)<t_{0}$. That is resolved by enforcing the condition,

$$
y_{d}(t)=\bar{y}\left(t_{0}\right), \quad \theta(y, t)<t_{0} .
$$

Away from events, system dynamics evolve smoothly according to the modified differential-algebraic model

$$
\begin{aligned}
\dot{x} & =f(x, y) \\
0 & =g\left(x, y, y_{d}\right) \\
y_{d}(t) & =\bar{y}(\theta(y, t))
\end{aligned}
$$

where $g$ is composed of $g^{(0)}$ together with appropriate choices of $g^{(i-)}$ or $g^{(i+)}$, depending on the signs of the corresponding elements of $y_{s}$.

System behaviour at switching and reset events is not directly influenced by time-delays. Rather, time-delays affect smooth behaviour between events, as described by (10)-(12). Therefore subsequent sections that explore implementation issues will focus on this modified DA system.

\subsection{Time-delay interpretation}

The general nonlinear form of the time-delay functional $\theta(y, t)$ in (3) places few restrictions on time-delay modelling. However for ease of implementation, it is convenient to assume causality, i.e.,

$$
\theta(y, t) \leq t .
$$

More traditional time-delay representations [5] fit within this general framework. For example a constant time-delay is simply

$$
\theta(y, t)=t-\theta_{c}
$$




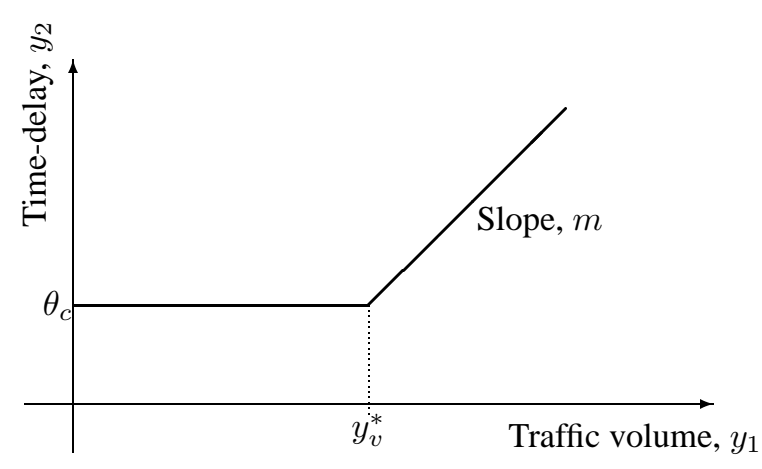

Fig. 2. Communications time-delay.

where $\theta_{c} \geq 0$ is constant.

As mentioned in Section 1, average time-delays over communications links are generally not constant, but rather have a form akin to Fig. 2. The average time-delay is relatively constant for low traffic volume. However above a certain channel threshold, the time-delay increases. Fig. 2 shows a linear increase; the exact form is not important from the modelling perspective. The DAIS model allows straightforward implementation of the Fig. 2 time-delay,

$$
\begin{array}{cc}
0=y_{2}-\theta_{c}, & y_{1}<y_{v}^{*} \\
0=y_{2}-\theta_{c}-m\left(y_{1}-y_{v}^{*}\right), & y_{1}>y_{v}^{*} \\
\theta(y, t)=t-y_{2} . &
\end{array}
$$

The generality of (3) does however raise some interesting technical issues. For example, it can be shown that simple structures such as

$$
y_{2}(t)=y_{1}\left(t-y_{2}\right)
$$

may result in non-uniqueness of solutions. Details are beyond the scope of this present paper.

\section{SIMULATION}

The modified DAIS model (1)-(4), or alternatively (10)(12) along smooth sections of trajectories between events, is only useful if a reliable numerical integration process can be established. Implicit integration techniques, such as BDF formulae or trapezoidal integration, are known to be robust for differential-algebraic systems [6]. We have adopted trapezoidal integration due to the relative simplicity of implementation.

Full details of trapezoidal integration of the DA model (10)-(12), with no time-delays, is provided in [4]. The focus here is on the modifications required to incorporate time-delays. Trapezoidal approximation of the differential equations (10) results in the coupled set of algebraic equa- tions

$$
\begin{aligned}
& 0=F_{1}(x, y) \\
& 0=g\left(x, y, y_{d}\right) \\
& 0=F_{2}\left(y, y_{d}\right)=y_{d}-\bar{y}(\theta(y, t))
\end{aligned}
$$

which must be solved for $x, y$ and $y_{d}$ at each time step. This is achieved using Newton's method, which involves building and inverting the Jacobian

$$
\mathcal{J}=\left[\begin{array}{ccc}
\frac{\partial F_{1}}{\partial x} & \frac{\partial F_{1}}{\partial y} & 0 \\
\frac{\partial g}{\partial x} & \frac{\partial g}{\partial y} & \frac{\partial g}{\partial y_{d}} \\
0 & \frac{\partial F_{2}}{\partial y} & \frac{\partial F_{2}}{\partial y_{d}}=I_{d}
\end{array}\right]
$$

where $I_{d}$ is the $d$-dimensional identity matrix. The only term of any real novelty is $\frac{\partial F_{2}}{\partial y}$, which can be obtained via the chain rule as,

$$
\frac{\partial F_{2}}{\partial y}=-\operatorname{diag}\left\{\frac{d \bar{y}}{d t}(\theta(y, t))\right\} \frac{\partial \theta}{\partial y}
$$

where $\operatorname{diag}\{$.$\} refers to a diagonal matrix built from the$ associated vector.

Due to the simple structure of $F_{2}$, it is convenient to solve explicitly for $y_{d}$ by substituting $\bar{y}$ directly into (14), resulting in the algebraic equation

$$
0=g(x, y, \bar{y}(\theta(y, t))) \text {. }
$$

The corresponding reduced Jacobian then has the form

$$
\mathcal{J}_{\text {red }}=\left[\begin{array}{cc}
\frac{\partial F_{1}}{\partial x} & \frac{\partial F_{1}}{\partial y} \\
\frac{\partial g}{\partial x} & \left(\frac{\partial g}{\partial y}+\frac{\partial g}{\partial y_{d}} \operatorname{diag}\left\{\frac{d \bar{y}}{d t}(\theta(y, t))\right\} \frac{\partial \theta}{\partial y}\right)
\end{array}\right] .
$$

Notice that time-delays really only alter the effective $\frac{\partial g}{\partial y}$, though that alteration can be significant.

Evaluation of the Jacobian $\mathcal{J}$, or alternatively $\mathcal{J}_{\text {red }}$, requires the computation of $\frac{d \bar{y}}{d t}(\theta(y, t))$, the rate of change of $\bar{y}$ at time $\theta(y, t)$, i.e., some time in the past. Therefore the numerical integration process requires evaluation (and storage) of $\frac{d \bar{y}}{d t}$ along the trajectory. To achieve this, differentiation of (11)-(12) with respect to $t$, along with some manipulation, yields

$$
\begin{aligned}
0=\frac{\partial g}{\partial x} f(x, y) & +\left[\frac{\partial g}{\partial y}+\frac{\partial g}{\partial y_{d}} \operatorname{diag}\left\{\frac{d \bar{y}}{d t}(\theta(y, t))\right\} \frac{\partial \theta}{\partial y}\right] \frac{d y}{d t} \\
& +\frac{\partial g}{\partial y_{d}} \operatorname{diag}\left\{\frac{d \bar{y}}{d t}(\theta(y, t))\right\} \frac{\partial \theta}{\partial t},
\end{aligned}
$$

from which $\frac{d y}{d t}$ may be obtained. If follows from (5) that

$$
\frac{d \bar{y}}{d t}(t)=\frac{d y_{[\ell]}}{d t}(t)
$$

where $\ell$ is the indexing vector. Implementation has assumed that

$$
\frac{d \bar{y}}{d t}(\theta(y, t))=0, \quad \text { for } \theta(y, t)<t
$$




\section{TRAJECTORY SENSITIVITIES}

Trajectory sensitivities approximate the variation of a trajectory resulting from (small) changes to parameters and/or initial conditions [7]. To obtain the sensitivity of the flows $\phi_{x}$ and $\phi_{y}$ to initial conditions $x_{0}$, the Taylor series expansions of (6)-(7) are formed. Neglecting higher order terms gives

$$
\begin{aligned}
& \Delta x(t)=\frac{\partial x(t)}{\partial x_{0}} \Delta x_{0} \equiv \Phi_{x}(t) \Delta x_{0} \\
& \Delta y(t)=\frac{\partial y(t)}{\partial x_{0}} \Delta x_{0} \equiv \Phi_{y}(t) \Delta x_{0} .
\end{aligned}
$$

These equations describe the changes $\Delta x(t)$ and $\Delta y(t)$ in a trajectory, at time $t$ along the trajectory, for a given (small) change in initial conditions $\Delta x_{0}$. The time-varying partial derivatives $\Phi_{x}$ and $\Phi_{y}$ are known as trajectory sensitivities. Complete details of the variational equations describing the evolution of these sensitivities for the DAIS model are provided in [4].

The introduction of time-delays into the DAIS model results in modification of the associated variational equations. The time-delay version is obtained by differentiating (11)-(12) with respect to $x_{0}$, resulting in

$$
\begin{aligned}
0=\frac{\partial g}{\partial x} \Phi_{x}(t) & +\left[\frac{\partial g}{\partial y}+\frac{\partial g}{\partial y_{d}} \operatorname{diag}\left\{\frac{d \bar{y}}{d t}(\theta(y, t))\right\} \frac{\partial \theta}{\partial y}\right] \Phi_{y}(t) \\
& +\frac{\partial g}{\partial y_{d}} \Phi_{\bar{y}}(\theta(y, t)) .
\end{aligned}
$$

The role of the modified $\frac{\partial g}{\partial y}$ is again evident.

\section{APPLICATIONS}

Incorporating time-delays into the DAIS model in a systematic way has maintained an efficient process for obtaining trajectory sensitivities as a by-product of numerically computing the trajectory. Trajectory sensitivities are effectively gradients of the trajectory, and so can be used to build gradient-based iterative algorithms.

Numerous applications follow, including parameter estimation, boundary value problems, and optimal control. For example, a shooting method [8] could be formulated to determine the critical value of a delay that induced oscillations. Work is proceeding in the development of such applications [3].

\section{CONCLUSIONS}

Modelling of multi-layer power system interactions will become increasingly important as market mechanisms and feedback controls become more tightly coupled into the physical system layer. Time-delays are an important aspect of that inter-layer coupling.

This paper has established a deterministic, nonlinear time-delay model, and incorporated that model into a systematic hybrid (continuous/discrete) system representation. It has been shown that time-delays affect the algebraic Jacobian of the differential-algebraic model. This modified Jacobian follows through into the computation of trajectory sensitivities.

Availability of trajectory sensitivities facilitates gradientbased iterative algorithms for solving inverse problems such as parameter estimation, boundary value problems, and optimal control.

\section{Acknowledgement}

The valuable assistance of Dr. Reginald Goatcabin is gratefully acknowledged.

\section{REFERENCES}

[1] F.L. Alvarado, J. Meng, C.L. DeMarco, and W.S. Mota, "Stability analysis of interconnected power systems coupled with market dynamics," IEEE Transactions on Power Systems, vol. 16, no. 4, pp. 695-701, November 2001.

[2] A. Tarantola, Inverse Problem Theory, Elsevier Science, Amsterdam, 1987.

[3] I.A. Hiskens, "Power system modeling for inverse problems," IEEE Transactions on Circuits and Systems I, Submitted.

[4] I.A. Hiskens and M.A. Pai, "Trajectory sensitivity analysis of hybrid systems," IEEE Transactions on Circuits and Systems I, vol. 47, no. 2, pp. 204-220, February 2000.

[5] Z.J. Palmor, "Time-delay compensation - Smith predictor and its modifications," in The Control Handbook, W.S. Levine (Editor), CRC Press, Boca Raton, FL, 1996.

[6] K.E. Brenan, S.L. Campbell, and L. Petzold, Numerical Solution of Initial-Value Problems in DifferentialAlgebraic Equations, SIAM, Philadelphia, PA, 1995.

[7] P.M. Frank, Introduction to System Sensitivity Theory, Academic Press, New York, 1978.

[8] T.S Parker and L.O. Chua, Practical Numerical Algorithms for Chaotic Systems, Springer-Verlag, New York, NY, 1989. 